\title{
¿Qué Piensan Estudiantes de Primaria Bonaerenses y Madrileños sobre la Participación Escolar?
}

\section{What do Primary School Students of Buenos Aires and Madrid Think about School Participation?}

\author{
Almudena Juanes * \\ Liliana Jacott \\ Universidad Autónoma de Madrid, España
}

\begin{abstract}
El presente estudio pretende conocer las ideas que tienen sobre participación escolar estudiantes de $4^{\circ}$ y $6^{\circ}$ curso de Educación Primaria de Madrid y Buenos Aires. Se realizan 153 entrevistas semiestructuradas individuales con niñas y niños de 9 y 11 años de ambos países en donde se les plantea resolver un dilema específico sobre participación democrática escolar. Los resultados muestran que chicas y chicos de $4^{\circ}$ y $6^{\circ}$ de Primaria de ambas ciudades mantienen mayoritariamente una concepción de la participación desde un enfoque en el que se destaca el papel de la autonomía personal en la toma de decisiones democráticas, en contraste con un enfoque más centrado en la autoridad y el mérito. Asimismo, se aprecian diferencias de género en las representaciones sobre la participación escolar en ambas muestras, al igual que diferencias por ciudades en dichas representaciones. Concluimos resaltando la importancia de indagar cómo se desarrollan y coordinan las distintas representaciones infantiles cuando interpretan y evalúan la realidad social a partir de sus propias experiencias con las prácticas culturales y contextos educativos particulares, para dar cuenta de la participación, la toma de decisiones y la justicia social.
\end{abstract}

Descriptores: Participación escolar; Justicia social; Educación democrática; Elecciones; Estudiantes de primaria.

The present study aims to find out the ideas that 4th and 6th grade Primary Education students in Madrid and Buenos Aires have about school participation. 153 individual semi-structured interviews were conducted with girls and boys aged 9 and 11 from both countries, in which they were asked to resolve a specific dilemma regarding democratic participation in schools. The results show that girls and boys in 4th and 6th grade of primary school in both cities mostly maintain a conception of participation from an approach that emphasizes the role of personal autonomy in democratic decision-making, in contrast to a focus more centered on authority and merit. There are also gender differences in the representations of school participation in both samples, as well as differences by city in these representations. We conclude by highlighting the importance of investigating how different children's representations develop and coordinate when they interpret and evaluate social reality based on their own experiences with particular cultural practices and educational contexts, in order to account for participation, decision-making and social justice.

Keywords: School participation; Social justice; Democratic education; Elections; Primary school students.

*Contacto:

almudena.juanes@educa.madrid.org

ISSN: 2254-3139

www.rinace.net/riejs/

revistas.uam.es/riejs
Recibido: $\quad 15$ de julio 2020

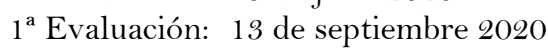

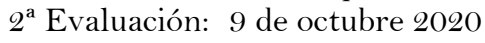

Aceptado: 1 de noviembre 2020 


\section{Introducción}

La participación escolar constituye un concepto controvertido con múltiples significados que ha sido abordado desde diferentes perspectivas educativas y con objetivos distintos. En algunos países se pretende que la participación democrática forme parte del día a día en la vida escolar. Mientras que en otros contextos socioculturales son muy escasas las experiencias de participación infantil y juvenil en los centros escolares. Desde un enfoque de educación para la ciudadanía se pretende que las personas jóvenes adquieran los conocimientos, capacidades y actitudes necesarias que les permitan desarrollar un papel activo, informado y crítico para participar en una sociedad democrática (García-Vélez, 2016). De modo que participación y democracia son dos componentes claves que han sido objeto de estudio desde variados enfoques. En la presente investigación, analizamos las representaciones que tienen estudiantes de Educación Primaria de dos países distintos sobre participación escolar. Y más concretamente, abordamos el estudio de la participación como una de las dimensiones de justicia social ${ }^{1}$ (Fraser, 2008a, 2008b; Miller, 1999). Desde nuestra perspectiva, consideramos a las tres dimensiones: Redistribución, Reconocimiento y Representación/Participación, como herramientas para poder aproximarnos a la Justicia Social (Murillo y Hernández, 2011), y entendemos esta, como un medio para que todas las personas alcancen el objetivo de tener una vida digna, en la que se promueva activamente el desarrollo de sus capacidades (Jacott et al., 2016a, 2016b, 2019).

En este estudio, como indicamos, nos centramos especialmente en la dimensión de participación, bajo la idea de la plena participación de todas las personas implicadas (estudiantes, profesorado, familias, comunidad educativa), en la toma de decisiones que tienen lugar en el proceso educativo, y más concretamente, la participación del alumnado. Haciendo especial hincapié en promover mecanismos de participación, en dar voz desde una visión transformadora al conjunto de estudiantes, como una parte muy importante de su proceso formativo como ciudadanas y ciudadanos del siglo XXI, para que puedan ser capaces de cuestionar, resistir o transformar los sistemas que promueven las injusticias y desigualdades (Pearce y Wood, 2019).

Es crucial que estas ideas sobre el rol que debe tener la educación desde el enfoque de la Justicia social se trabajen tanto desde las escuelas, como desde la investigación, escuchando todas las voces implicadas, con el objetivo de determinar cuál es el impacto que tienen estas prácticas en educación y cómo son comprendidas y abordadas las situaciones de injusticia y desigualdad en los entornos educativos por parte de estudiantes, profesorado, familias y comunidad educativa. Y para conseguirlo es necesario diseñar herramientas que nos permitan conocer con mayor profundidad cuáles son sus ideas acerca

\footnotetext{
${ }^{1} \mathrm{El}$ presente estudio forma parte de un trabajo más amplio, donde se investigan las ideas del alumnado en las tres dimensiones de Justicia Social. Se enmarca en los siguientes proyectos: "Escuelas para la Justicia Social" (2012-2014), EDU2011-29114, y Proyectos de Investigación Científica y Desarrollo Tecnológico, I+D+I Ministerio de Ciencia e Innovación, Convocatoria 2011 de ayudas para la realización de Proyectos de Investigación Fundamental no orientada; "Educación y Justicia Social: una mirada multidisciplinar" (2012-2014), CEMU-2012-024, I Convocatoria de Proyectos de Investigación Multidisciplinares de la UAM y "Escuelas en contextos socio-económicamente desafiantes: Una aproximación desde la educación para la Justicia Social” (2015-2018), EDU2014-56118-P.
} 
de la justicia social, y más concretamente, en el caso que nos ocupa, sobre la participación en contextos educativos.

\section{Revisión de la literatura}

En cuanto a los estudios sobre participación escolar, cabe señalar la existencia de una serie de trabajos relevantes realizados en distintos países en los que abordan este tema desde variados enfoques teóricos y metodológicos. Así por ejemplo, encontramos investigaciones en los que se aborda la participación desde enfoques más centrados en educación para la ciudadanía (Holdsworth, 2000), las voces de estudiantes en la toma de decisiones de la propia escuela (Cook-Shater, 2020; Granizo, 2011; Pearce y Wood, 2019; Thornberg y Elvstrand, 2012), la importancia de la participación como elemento de mejora de la escuela (Lodge, 2005; Rudduck y Fielding, 2006), la participación de estudiantes y familias en la toma de decisiones en distintos contextos de su vida cotidiana (Seguro, 2016), o desde la perspectiva de estudiantes con necesidades educativas especiales (Koster et al., 2010).

En cambio, parece que aún hay pocos trabajos que cuenten con instrumentos validados que midan la participación de estudiantes en decisiones que afectan a sus vidas (O'Hare et al., 2016), siendo estos en su mayoría cuantitativos, frente a los cualitativos (Mager y Nowak, 2012; O’Hare et al., 2016) y en muchas ocasiones partiendo de las ideas de las personas adultas, con respecto a la participación de sus hijas e hijos (Horgan et al., 2017; O’Hare et al., 2016; Sales et al., 2018), o de las del profesorado con respecto a la participación de sus estudiantes (Heikkila, Vuopala y Leinonen, 2017; Sales, et al., 2018), o en relación con sus prácticas pedagógicas (Hidalgo y Perines, 2018).

El reconocimiento al derecho de participación de estudiantes, especialmente en la infancia, ha sido planteado desde hace años, tanto desde algunos trabajos e investigaciones pioneras en este campo (Dewey, 1916; Melton, 1980), como desde organizaciones internacionales como la ONU a través de la CDN (Convención de las Naciones Unidas sobre los Derechos del Niño, 1989). De hecho, en los últimos años se han realizado una serie de estudios relevantes sobre los derechos de participación en la infancia y adolescencia (Burger, 2017; Campos, 2015; García-Pérez y Montero, 2014; Giné et al., 2014; Ochaíta y Espinosa, 2004; Robles, 2015; Ros, 2009; Seguro, 2016; Susinos y Rodríguez-Hoyos, 2011).

Sin embargo, encontramos que hay un menor número de estudios sobre participación en el ámbito educativo en los que se analicen las ideas que tienen los propios estudiantes acerca de su participación en la escuela (Coiduras et al., 2016) o sobre la percepción de sus derechos (Burger, 2017; Seguro, 2016). Y menos aún se encuentran estudios sobre las representaciones de estudiantes desde una perspectiva de las tres dimensiones de Justicia social (Juanes, 2018; Sainz, 2017).

Si atendemos al nivel educativo, encontramos que son más escasas las investigaciones realizadas en Educación Primaria (García-Pérez y Montero, 2014; Giné et al., 2014), frente a la Educación Secundaria (Goodnight, Whitley y Brophy-Dick, 2019; Granizo, 2011; Grayman y Godfrey, 2013; Mager y Nowak, 2012; De Smul et al., 2019).

A partir del meta-análisis llevado a cabo por Mager y Nowak (2012) sobre los efectos de la participación estudiantil en la toma de decisiones escolares, puede decirse que un buen número de estudios empíricos realizados en este ámbito se centran en la participación del alumnado dentro de la propia escuela, fundamentalmente a través de consejos escolares y 
toma de decisiones en la clase, e incluso en la planificación, implementación y evaluación de procesos de enseñanza-aprendizaje (Cook-Shater, 2020; De Smul et al., 2019; Goodnight et al., 2019; Hidalgo y Perines, 2018; Heikkila, Vuopala y Leinonen, 2017), no tratándose la participación del alumnado en temas que trascienden la propia escuela, como las injusticias y desigualdades sociales.

Además, hay que destacar que buena parte de las investigaciones que se encuentran en este ámbito son llevadas a cabo en países como Irlanda, Reino Unido, Estados Unidos o Canadá (Horgan et al., 2017; Mager y Nowak, 2012; Goodnight et al., 2019; Pearce y Wood, 2019) siendo más escasos los trabajos realizados en otros países, y que comparen grupos de edad, países, o contextos diversos, y en el caso de dichas comparaciones, no parece haber resultados concluyentes (Mager y Nowak, 2012; O’Hare et al., 2016)

Se han hallado numerosos beneficios de la participación del alumnado en los centros educativos, como: mejora de autoestima y relaciones alumnado-docentes (Mager y Novwak, 2012; O’Hare et al., 2016), habilidades para la resolución de problemas, (Mager y Nowak, 2012), mejora del aprendizaje (De Smul et al., 2019; Goodnight et al., 2019), rendimiento académico en la escuela (Ros, 2009) y del funcionamiento de la escuela (Lodge, 2005; Rudduck y Fielding, 2006), entre otros.

En cambio, resulta necesario atender a las condiciones en las que se promueve dicha participación, de forma que influya en las políticas reales de las escuelas, y no se perciba por parte de los estudiantes como algo externo y ajeno a sus vidas (Bruno y Barreiro, 2015; Grayman y Godfrey, 2013) produciendo desilusión (Mager y Nowak, 2012), o resultando un comportamiento moldeado por los adultos (Coiduras et al., 2016; Thornberg y Elvstrand, 2012).

Si bien los derechos de participación en la infancia son reconocidos a nivel global (Convención de las Naciones Unidas sobre los Derechos del Niño, 1989), parece que todavía en la actualidad existe una falta de reconocimiento social y educativo de la infancia como una etapa donde los niños deben ser vistos como personas activas en la sociedad, por lo que deberían ser tratadas como ciudadanas y ciudadanos del presente y no del futuro. Al igual que existe un interesante debate sobre cuál es el alcance real de los derechos de participación y de lo que significa la participación escolar, tanto a nivel conceptual como en las prácticas pedagógicas (Burger, 2017; Coiduras et al., 2016; Larkins, 2014; Percy-Smith 2010, 2015; Thornberg y Elvstrand, 2012).

Así, el concepto de participación en la infancia constituye un concepto controvertido y que plantea muchos problemas difíciles de resolver ya que, por ejemplo, todavía en la actualidad se cuestiona por parte de las personas adultas cuáles son las edades y cuál es el rango de actividades y escenarios en que los niños pueden ser capaces de participar para tomar decisiones en su vida cotidiana. No obstante, algunos autores como Burger (2017) comienzan a apuntar una serie de recursos sociales y psicológicos, para que se dé mayor participación en el alumnado, tales como un clima percibido de no discriminación, bienestar subjetivo y sentimientos de seguridad en el alumnado, o tener personas de confianza en los centros escolares.

Igualmente, tal como plantean recientemente Horgan y otros (2017), todavía son pocos los estudios en los que se abordan las experiencias concretas que tienen los niños sobre sus derechos de participación, o en los que se analiza su participación real y efectiva en los 
distintos contextos cotidianos relacionales en los que se desarrollan y cuáles son las condiciones necesarias para ello.

Algunos de estos trabajos que analizan su participación relacional en distintos escenarios de la vida cotidiana, nos muestran que aquellos escenarios en los que es más frecuente que se promueva su voz y participación suelen ser su propio hogar o asociaciones pequeñas. En contraste con su escuela o la comunidad, como estructuras más formales de participación, en las que la participación infantil se encuentra más limitada tanto por las actitudes de personas adultas como por el significado que dan a una serie de elementos relacionados con la edad, voz y capacidades necesarias para participar (Horgan et al., 2017).

De hecho, las oportunidades que tiene la infancia para participar en los espacios de la esfera pública suelen ser muy limitadas, y esto es algo que ocurre particularmente en las escuelas (Horgan et al., 2017). Muchos jóvenes se muestran poco satisfechos con su nivel de participación en los procesos de toma de decisiones en la escuela y tienen nivel muy bajo de expectativas de que las escuelas sean sitios de participación salvo en algunas pocas cuestiones periféricas en la escuela (Horgan et al., 2017; Thornberg y Elvstrand, 2012).

Así que más bien, tendríamos que centrarnos en pensar la participación como un conjunto más amplio y diverso de procesos sociales, poniendo el foco en los entornos cotidianos y en las interacciones en los que ésta tiene lugar (Percy-Smith, 2010, 2015). Y sobre todo en analizar cuáles son las prácticas cotidianas que se dan en la infancia en las distintas esferas de sus vidas y cómo interpretan sus propias prácticas de participación en los distintos contextos en que se producen (Larkins, 2014). Junto a ello, pensamos que es necesario abordar con mayor profundidad el estudio de las representaciones que tienen estudiantes de distintos contextos socioculturales sobre las prácticas de participación.

Teniendo en cuenta lo expuesto hasta aquí, en el presente estudio partimos de la problemática existente en cuanto a las ideas de participación infantil, ya que por un lado, parece que se da poca participación infantil real en las escuelas y por otro, no siempre se entiende a la infancia como una etapa en la que las personas puedan participar de forma plena como ciudadanos del presente, siendo críticos con las injusticias sociales, y con una clara vinculación a la comprensión de dicha participación desde la perspectiva de la justicia social.

Es por ello que, en nuestra investigación, nos planteamos el siguiente objetivo general: conocer las ideas sobre participación escolar que tienen estudiantes de $4^{\circ}$ y $6^{\circ}$ de Educación Primaria de Madrid y Buenos Aires, cuando resuelven un dilema de justicia social acerca de uno de los procesos democráticos de toma de decisiones en la escuela: la elección de un delegado o representante de clase.

Así pues, de forma más concreta, nuestros objetivos específicos son:

- Averiguar las ideas del alumnado respecto a la participación en contextos cotidianos como la escuela. Nos interesa por un lado saber cuáles son sus ideas acerca de los distintos mecanismos o procedimientos que pueden seguirse para la elección de un delegado de clase. Y por otro, indagar cuáles son sus ideas acerca del papel que tienen las figuras de autoridad (en este caso, la profesora) en el proceso de elección de representante escolar, cómo entienden las normas dadas en las escuelas (aceptación o rechazo de las normas impuestas, crítica o 
asunción de estas, si las consideran justas o injustas, etc.) y la importancia que otorgan al criterio del mérito académico en dicho proceso.

- Conocer las diferencias que existen a partir del análisis de las argumentaciones y justificaciones dadas por estudiantes, en función del grado en que sus representaciones sobre la participación se posicionan como más o menos cercanas a una perspectiva de Justicia Social, de manera que nos permita formar distintos niveles de complejidad en las representaciones, desde este enfoque.

- Establecer posibles comparaciones por curso escolar, género y ciudad respecto a las ideas sobre participación escolar generadas a partir del dilema planteado.

\section{Método}

Se realiza un estudio exploratorio de tipo cualitativo, descriptivo, con un diseño transversal (Bernard, Wutich y Ryan, 2017). Para el estudio cualitativo se diseña una entrevista semiestructurada para ser aplicada de forma individual. Una vez diseñada, la entrevista fue validada a partir de la realización de un estudio piloto con una muestra total de 20 estudiantes de Educación Primaria de Madrid (seleccionados proporcionalmente, por género y curso). Los resultados del estudio piloto mostraron que la entrevista resultó un instrumento útil de evaluación, permitiendo establecer comparaciones entre las ideas del alumnado de Educación Primaria, según el curso y el género (Juanes, Pérez-Manjarrez y Fernández-González, 2013). La entrevista definitiva presenta seis dilemas, dos para cada dimensión de justicia social.

\section{Participantes}

La muestra consta de 153 estudiantes de cuatro escuelas públicas de Buenos Aires y Madrid. En el cuadro 1 se indica la muestra total de estudiantes, por ciudad, curso y género. De la muestra total, el $48 \%$ de estudiantes pertenecen a centros educativos argentinos y el $52 \%$ a centros españoles. En cuanto al curso, $49,7 \%$ son estudiantes de $4^{\circ}$ y $50,3 \%$ de $6^{\circ}$ de Educación Primaria. Y con respecto al género, 49,7\% son niñas y 50,3\% son niños. Las edades medias del alumnado son: para $4^{\circ}$ curso de 9,6 años (Buenos Aires) y 9,2 años (Madrid), y para $6^{\circ}$ curso de 1 1,4 años (Buenos Aires) y 11,1 años (Madrid).

Cuadro 1. Muestra del estudio

\begin{tabular}{llccc}
\hline & & BUENOS AIRES & MADRID & TOTAL \\
\hline \multirow{3}{*}{$4^{\circ}$ Primaria } & Niñas & 19 & 20 & 39 \\
\cline { 2 - 5 } & Niños & 17 & 20 & 37 \\
\cline { 2 - 5 } & Total & 36 & 40 & 76 \\
\hline \multirow{3}{*}{$6^{\circ}$ Primaria } & Niñas & 19 & 18 & 37 \\
\cline { 2 - 5 } & Niños & 18 & 42 & 40 \\
\cline { 2 - 5 } Total & Total & 37 & 38 & 77 \\
\hline Total participantes & Niñas & 38 & 42 & 77 \\
\cline { 2 - 5 } & Niños & 35 & 80 & 153 \\
\hline
\end{tabular}

Fuente: Elaboración propia.

La selección de las escuelas se realizó teniendo en cuenta fundamentalmente dos criterios. El primero de ellos vinculado a la justicia social, de forma que se pudiera contar con centros escolares de dos tipos: escuelas que como parte de su proyecto pedagógico y educativo fuesen de alguna manera más favorecedoras de la justicia social y otros centros 
de tipo más estándar, que no fueran en principio especialmente promotores de la justicia social. Y el segundo de ellos se intentó que fuesen centros escolares que estuviesen situados en un contexto socioecónomico medio y bajo.

En el caso de Buenos Aires, las cuatro escuelas forman parte de la zona del conurbano bonaerense que rodea la capital, siendo dos las que consideramos como más promotoras de la justicia social, y dos las escuelas estándar. En cuanto a la ciudad de Madrid, escogimos dos escuelas como más favorecedoras de la justicia social, situadas en la zona sureste de la capital, y dos escuelas de tipo más estándar, localizadas en la zona norte.

Cabe señalar que, en el presente trabajo, ofrecemos una visión general de la opinión del alumnado en ambos países, y los argumentos que ofrecen niñas y niños para justificar sus decisiones más o menos justas. Por ello, no exponemos aquí las respuestas de los estudiantes en función de cada escuela a la que pertenecían, sino a nivel global, con relación a su edad, género o ciudad.

\section{Instrumento}

La entrevista es semiestructurada y consta de seis dilemas sobre distintas situaciones relacionadas con la justicia social. Si bien en este estudio solo nos centraremos en analizar los resultados de uno de los dilemas. En estos dilemas, se exponen diversos escenarios de injusticia, y se pide al alumnado que sugiera qué haría ante estas situaciones, o cómo estas podrían resolverse, de modo abierto, sin ofrecer ninguna alternativa de respuesta. En este caso el dilema planteado corresponde a la dimensión de Participación denominado: "Elección de representante" (cuadro 2).

Cuadro 2. Guion del dilema

\begin{tabular}{lc}
\hline \multicolumn{1}{c}{ DESCRIPCIÓN } & MINUTOS \\
\hline Apertura inicial y presentación & 3 \\
\hline Consentimiento informado y confidencialidad & 3 \\
\hline Presentación del dilema: Elección de representante \\
“Hay delegados/delegadas, representantes de estudiantes, en tu clase?, ̇̇abes lo que son?” \\
(Si en su escuela no ha habido nunca un representante de la clase o si desconocen \\
lo que significa el término "delegado”, se explica en qué consiste esta figura). \\
“..La semana pasada estuve en una escuela y en un grupo estaban decidiendo cómo elegir \\
a un estudiante como delegada o delegado para que fuera su representante y Paula \\
(estudiante de $4^{\circ} / 6^{\circ}$ ) le dijo a su maestra que ella quería ser, pero la profesora decía que \\
no, que debería ser delegada otra estudiante que tuviera mejores notas, porque Paula \\
quiere ser delegada pero no saca muy buenas notas. \\
¿A ti qué te parece? ¿Crees que es importante sacar buenas notas para poder ser \\
representante de tus compañeras y compañeros de clase, para ser delegada/delegado o \\
no? \\
¿Qué debería tener o cómo debería ser una estudiante para poder ser representante? \\
(Dependiendo de las respuestas generadas por cada estudiante, la entrevistadora \\
realiza distintas contra sugerencias. Por ejemplo, si un alumno indica que en su \\
grupo se hace mediante votación, la entrevistadora pregunta qué le parece esa \\
manera de proceder, si es mejor o peor de lo que se plantea en el dilema, o si lo \\
haría de otra forma).
\end{tabular}

\section{Procedimiento}

Las entrevistas, se llevaron a cabo de forma individual, en una pequeña sala, cercana al aula de referencia, tanto en las escuelas madrileñas como en las bonaerenses. En todos los casos, se contó con el consentimiento informado del equipo directivo, familias y 
estudiantes. Las entrevistas tuvieron una duración de aproximadamente 10 minutos para este dilema, y se grabaron y transcribieron en su totalidad por la primera autora, para su análisis ulterior.

\section{Análisis de los datos}

Para examinar los resultados de las entrevistas, se realiza un análisis de contenido de las respuestas al dilema (Braun y Clarke, 2006). En este análisis, se identifican posibles categorías a partir de la lectura de las transcripciones de las entrevistas. Al igual que en otras investigaciones, las categorías no estaban predeterminadas, sino que surgieron de forma inductiva a partir de las respuestas generadas por los participantes (Mager y Nowak, 2012).

En un primer momento establecemos tres categorías distintas de respuesta en función de cómo se resuelve el dilema en términos de la importancia que se otorga a los siguientes elementos: el procedimiento que utilizan para la elección de representante, el papel que dan a la figura de autoridad (la profesora), las normas de la escuela y el mérito personal en este proceso de elección de representante escolar. En este caso las categorías son:

- participación desde una perspectiva centrada en la autoridad y el mérito.

- participación desde una perspectiva de autonomía personal.

- participación desde una perspectiva de justicia distribuida como equidad.

Y en un segundo momento, categorizamos estos tres tipos de participación en función del nivel de posicionamiento que tiene la solución del dilema con respecto a un enfoque de justicia social, en la dimensión de participación. En el cuadro 3 se recogen los distintos niveles de justicia social asociados a los tipos de participación con su descripción y categorías temáticas correspondientes.

Como puede verse en el cuadro 3, agrupamos las respuestas en tres categorías, situándolas bajo un continuo que va del nivel I al nivel III, de respuestas poco vinculadas a la justicia social y más concretamente a la dimensión de participación (nivel I), a respuestas más relacionadas con la justicia social y la participación en distintos niveles (niveles II y III).

Las distintas categorías han sido creadas a partir de la clasificación de las respuestas dadas por los participantes, pero partiendo de distintos estudios en este ámbito. En este caso, al tratarse de cuestiones relacionadas con la comprensión de las normas y la justicia, nos basamos en investigaciones sobre el desarrollo de juicios morales en distintos contextos y situaciones (Neff y Helwig 2002, Nucci, 2001; Nucci, Turiel y Roded, 2018; Piaget, 1932; Turiel, 2002) y en los estudios sobre la ontogénesis de las representaciones sociales de justicia, creencia en un mundo justo y desarrollo de las justificaciones del castigo (Barreiro, 2012, 2013a, 2013b).

\section{Resultados}

\subsection{Niveles de participación}

\subsubsection{Nivel I: Participación desde un enfoque centrado en la autoridad y el mérito}

En esta categoría, se muestra una concepción de la participación desde un enfoque centrado en la toma de decisiones por parte de la autoridad, esto es, chicas y chicos admiten que sea únicamente la figura de autoridad, es decir, la profesora la que decida y no ofrecen 
ninguna otra alternativa más democrática para la elección del delegado. Las razones o motivos utilizados para justificar sus respuestas son fundamentalmente el hecho de que la profesora es la persona que conoce mejor a sus estudiantes y, por tanto, tiene poder absoluto de decisión acerca de quién es el estudiante más acorde al cargo, atendiendo en este caso únicamente al criterio del mérito académico definido previamente por ella.

Cuadro 3. Niveles y tipos de participación

\begin{tabular}{|c|c|c|c|}
\hline Nivel & TIPO & DESCRIPCIÓN & $\begin{array}{c}\text { CATEGORÍAS } \\
\text { TEMÁTICAS }\end{array}$ \\
\hline Nivel I & $\begin{array}{l}\text { Participación } \\
\text { desde una } \\
\text { perspectiva } \\
\text { centrada en la } \\
\text { autoridad y el } \\
\text { mérito }\end{array}$ & $\begin{array}{l}\text { Las normas y procedimientos para elegir } \\
\text { representante escolar son impuestas por } \\
\text { agentes externos y dependen } \\
\text { básicamente de las decisiones y } \\
\text { perspectivas de las figuras de autoridad } \\
\text { y de criterios como el mérito. La } \\
\text { profesora es vista como una figura de } \\
\text { autoridad cuyas decisiones no pueden } \\
\text { ser cambiadas. Junto a ello, se valora la } \\
\text { importancia del mérito personal como } \\
\text { criterio para la toma de decisiones. }\end{array}$ & $\begin{array}{l}\text { Autoridad, apoyo } \\
\text { de toma de } \\
\text { decisiones por } \\
\text { parte de } \\
\text { personas adultas } \\
\text { Apoyo a sistemas } \\
\text { jerárquicos de } \\
\text { organización } \\
\text { social } \\
\text { Visión centrada en } \\
\text { el mérito } \\
\end{array}$ \\
\hline Nivel II & $\begin{array}{l}\text { Participación } \\
\text { desde una } \\
\text { perspectiva } \\
\text { autónoma }\end{array}$ & $\begin{array}{l}\text { Las normas y procedimientos para elegir } \\
\text { representante escolar son interpretados } \\
\text { desde una perspectiva más centrada en } \\
\text { la autonomía individual y por tanto } \\
\text { pueden ser modificadas. Se asume que la } \\
\text { decisión de la profesora puede ser } \\
\text { cuestionada y modificada según } \\
\text { criterios personales y grupales. }\end{array}$ & $\begin{array}{l}\text { Autonomía } \\
\text { personal } \\
\text { Visión más } \\
\text { democrática de } \\
\text { toma de } \\
\text { decisiones }\end{array}$ \\
\hline Nivel III & $\begin{array}{l}\text { Participación } \\
\text { desde una } \\
\text { perspectiva de } \\
\text { justicia } \\
\text { distributiva, } \\
\text { como equidad }\end{array}$ & $\begin{array}{l}\text { Las normas y procedimientos para elegir } \\
\text { representante escolar pueden ser } \\
\text { interpretadas desde distintas } \\
\text { perspectivas y por tanto pueden ser } \\
\text { modificadas. Se asume que la decisión } \\
\text { de la profesora puede ser cuestionada y } \\
\text { modificada según criterios democráticos } \\
\text { que buscan promover la equidad, la } \\
\text { solidaridad y el bienestar de otras } \\
\text { personas. Pueden incluirse acciones } \\
\text { conjuntas de participación para cambiar } \\
\text { una norma considerada injusta. }\end{array}$ & $\begin{array}{l}\text { Autonomía } \\
\text { centrada en } \\
\text { derechos } \\
\text { Participación } \\
\text { igualitaria en } \\
\text { toma de } \\
\text { decisiones } \\
\text { Visión centrada en } \\
\text { la búsqueda de } \\
\text { la equidad, } \\
\text { justicia, } \\
\text { solidaridad y } \\
\text { bienestar }\end{array}$ \\
\hline
\end{tabular}

Fuente: Elaboración propia.

En ocasiones, el alumnado formula un método alternativo para la elección de representante, como si se tratase de un pensamiento genuino, pero que, por el contrario, está estrechamente relacionado con el hecho de asumir la norma ya establecida: elección en función de los méritos académicos, entre los que se incluyen la realización de pruebas de distintas asignaturas a las personas candidatas, la revisión de las calificaciones, o la votación únicamente a estudiantes con óptimas calificaciones. Seguidamente se exponen dos respuestas de niños, como ejemplos de esta categoría:

Diego, $4^{\circ}$ Madrid: "Pues tiene un poquito de razón, (porque si saca malas notas...ya estamos empezando mal, entonces ¿̇cómo vas a ser un delegado?, si sacas malas notas... ¿̇cómo entiendes lo que te quiere decir la profe, si eres delegado?...) (Toma el papel como si fuera el profesor) To vería los cuadernos, o les diría unas cosas a cada uno de los compañeros, pero en bajito, lo mismo todos, como una pregunta, y ver cuál ha sido la mejor respuesta, y esa podría ser la delegada. (...). También se lo diría a 
la profe de inglés, para que ella también preguntara a cada uno... y que viera la mejor respuesta, para que también hubiera delegada en inglés, y tiene que saber mucho inglés (...). Haríamos preguntas de todo, de science, de...vería todas las respuestas y nos reuniríamos los profesores y hablaríamos, a ver cuál es la mejor (...) si están todos de acuerdo..."

Santino, $6^{\circ}$ Buenos Aires: "Sí, la maestra tiene razón, (si va mal en la escuela, ¿no?, no podés ser delegado, porque todos los compañeros sacan buenas notas y todo eso...porque si no sabes nada y todo eso, y sos delegado, alguno de los compañeros habla de un tema y vos no podés ni opinar de ese tema y eso sería malo... uno que saca buenas notas, sí, porque te puede ayudar, pero si sos delegado, es como que sos el jefe y no podés preguntar), entonces... sería mejor como dijo la señorita. To lo haría un concurso, primero empezando con matemáticas, quien sabe mejor de matemáticas, y después asi todas las materias... y el que saca mejor todas las notas...es, y esas notas también para el boletín, sería una prueba para todo, como un regalo”.

\subsubsection{Nivel II: Participación desde un enfoque autónomo}

En esta categoría, encontramos un avance en cómo interpretan la participación los estudiantes, pasando de una participación con un enfoque centrado en la autoridad y el mérito (nivel I), a una participación con un enfoque más autónomo a nivel personal y grupal. Por tanto, aquí las respuestas hacen alusión a métodos más participativos y democráticos de elección que implican contar con la capacidad de decisión y criterio de cada estudiante, distintos a los impuestos por la profesora, como, por ejemplo: el voto, donde todos participan y pueden ser elegidos. A continuación, se muestran ejemplos de respuestas en esta categoría:

Asier, $4^{\circ}$ Madrid: "Me gusta la forma que hacemos en mi clase, por votación entre todos, porque así no podemos decir: "jo, yo quiero, no es justo" o... Da igual que tenga malas notas o no, si te votan a ti... yo fui delegado el año pasado."

Abril, $6^{\circ}$ Buenos Aires (su hermana es delegada en secundaria): "En mi hermana, creo que pusieron una cajita y escribian el nombre del que querían y votaban todos los alumnos... la seño, no... Sí, está buena esa idea, a mí me gustaría hacerlo así, estaría bueno que votara también la seño. A mí me gustaría ser delegada en mi clase.”

\subsubsection{Nivel III: Participación desde un enfoque de justicia distribuida como equidad}

En esta última categoría plena de la dimensión de participación, desde un enfoque de justicia social, no solo se hace alusión a procedimientos más participativos y democráticos de elección que implican tener en cuenta la decisión de cada estudiante a partir de una votación, sino que al mismo tiempo se añaden mecanismos de participación nuevos en los que se tienen en cuenta distintos elementos de equidad durante el proceso. Y junto a ello encontramos argumentos que reflejan el deseo de que exista una mayor equidad, justicia, solidaridad y bienestar entre sus compañeras y compañeros de clase. En ocasiones, como método para asegurar que todos los estudiantes puedan ser delegados en algún momento en el curso escolar, mediante por ejemplo la alternancia de cada estudiante. Y en otras, para rebelarse de algún modo contra la norma impuesta, por considerarla injusta, logrando que en el dilema planteado Paula pueda ser representante de su grupo, independientemente de su rendimiento académico. En los siguientes ejemplos, encontramos este tipo de respuestas:

Marina, $6^{\circ}$ Madrid: "Hacerlo como lo hacemos nosotros con los encargados de asambleas... (van rotando y cada semana es una persona de la clase), porque así serían todos y no tiene que estar la profesora diciendo tú no porque no, tú tampoco...lo podemos hacer entre nosotros" 
Sira, $4^{\circ}$ Buenos Aires: "Pondría a la nena que no sabe, (Paula) para ayudarla a enseñar, y así ella se puede sentir bien y como que ya está más orgullosa así, como que ya se pone las pilas y así puede estudiar mejor, para que a ella no le de vergüenza ..."

Cristian, $6^{\circ}$ Madrid: "Pues no sé (...). Haríamos que firmara toda la clase, para decirle a la profesora que podría ser Paula la delegada, y así sabría que todos queremos que sea Paula (...) (Vale, ¿̇y en tu clase, los encargados cómo los elegís?) Son dos encargados, y cada dos semanas, van cambiando (...) (¿) $Y$ te parecería bien que en la clase de Paula lo hicieran asi??) "Si quisieran más personas ser delegados, sí, sino que fuera Paula".

Mateo, $6^{\circ}$ Madrid: "En mi clase los encargados no son los que sacan malas o buenas notas, depende del número de la lista, yo lo vería más justo, dos semanas dos delegados, otras dos, otros..."

\subsection{Análisis de los niveles de participación}

Cuando se analizan los porcentajes de respuesta obtenidos por la muestra total de participantes por nivel de participación se obtienen los siguientes resultados, tal como puede observarse en la figura 1.

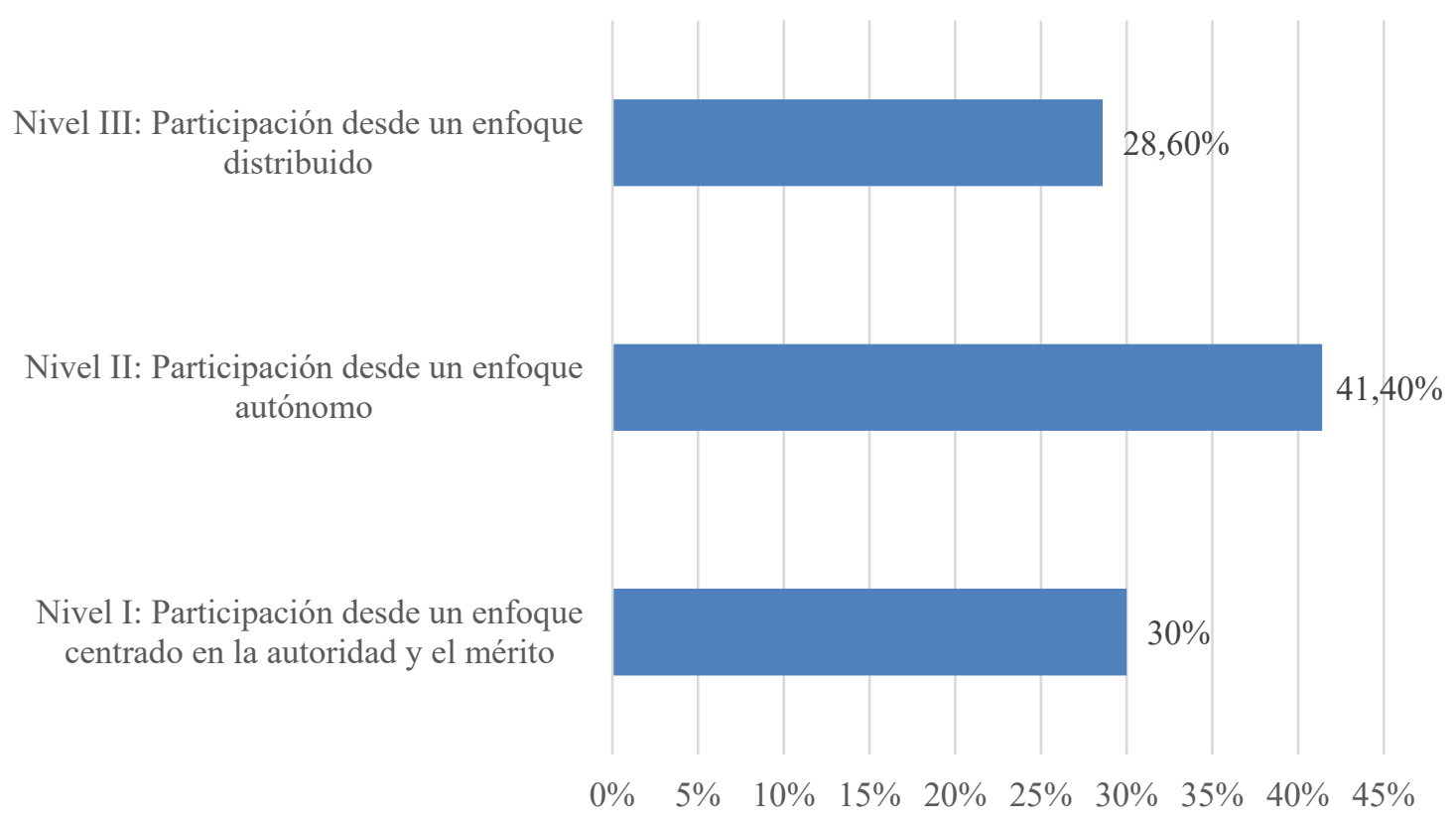

Figura 1. Niveles de participación en el dilema "Elección de representante de clase"

Fuente: Elaboración propia.

En la figura 1 observamos que el mayor número de respuestas del total de participantes de la muestra se halla en el nivel II (41,4\%), encontrando un porcentaje de 58,6 \% repartido entre los niveles I y III, con porcentajes muy similares $(30 \%$ y $28,6 \%$, respectivamente).

\subsection{Comparaciones por nivel educativo, género y ciudad}

Para realizar las comparaciones por nivel educativo, género y ciudad, se realizan pruebas $\chi^{2}$, encontrando diferencias estadísticamente significativas por género $(\mathrm{p}<0,05)$ y ciudad $(\mathrm{p}<0,00)$, pero no por curso $\left(\chi^{2}{ }_{2}=1,213 ; \mathrm{p}>0,05\right)$ 
En cuanto al género, los resultados muestran la existencia de diferencias significativas $\left(\chi^{2}{ }_{2}\right.$ $=6,355, \mathrm{p}=0,042)$, obteniendo las chicas puntuaciones más altas en el nivel III, con un porcentaje de $38,22 \%$, en comparación con los chicos (19,44\%). Por su parte, son los chicos quienes obtienen puntuaciones más altas en los niveles I y II, tal como se observa en la figura 2.

Igualmente, se muestra una tendencia decreciente en términos de mayor a menor justicia social en los niveles de participación alcanzados por las chicas en este estudio, obteniendo un porcentaje mayor de respuestas del nivel III (38,23\%), seguido por las del nivel II $(33,82 \%)$, y en menor medida por las del nivel I (27,94\%). En contraste con los chicos, cuyas respuestas siguen un patrón distinto, situándose en mayor proporción en el nivel II $(48,61 \%)$, después en el nivel I (31,94\%), siendo las de nivel III $(19,44 \%)$ las respuestas que obtienen una proporción más baja.

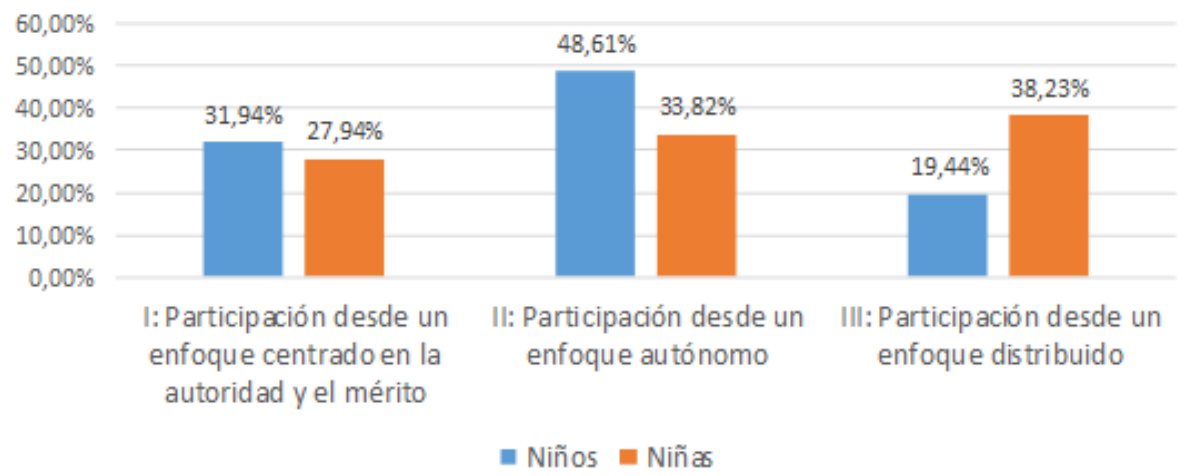

Figura 2. Niveles de participación por género Fuente: Elaboración propia.

$\mathrm{Y}$ con relación a la variable ciudad, se obtienen diferencias significativas $\left(\chi^{2}{ }_{2}=24,489, \mathrm{p}\right.$ $=0,00)$. Como podemos ver en la figura 3 , el alumnado madrileño obtiene un mayor porcentaje de respuestas en el nivel III $(44,90 \%)$ en comparación con el alumnado bonaerense $(8,1 \%)$, que obtiene mayores porcentajes en los niveles II $(48,4 \%)$ y I $(43,5 \%)$. Asimismo, las respuestas de los estudiantes madrileños ocupan el nivel III en un mayor porcentaje, seguidas del nivel II (35,9\%), siendo las de nivel I (19,2\%) las que menos proponen. En contraste, las respuestas de los estudiantes bonaerenses se sitúan en su mayoría en el nivel II (48,4\%), seguidas por las del nivel I (43,5\%) y siendo las de nivel III las respuestas que menos señalan, con un porcentaje de $8,1 \%$.

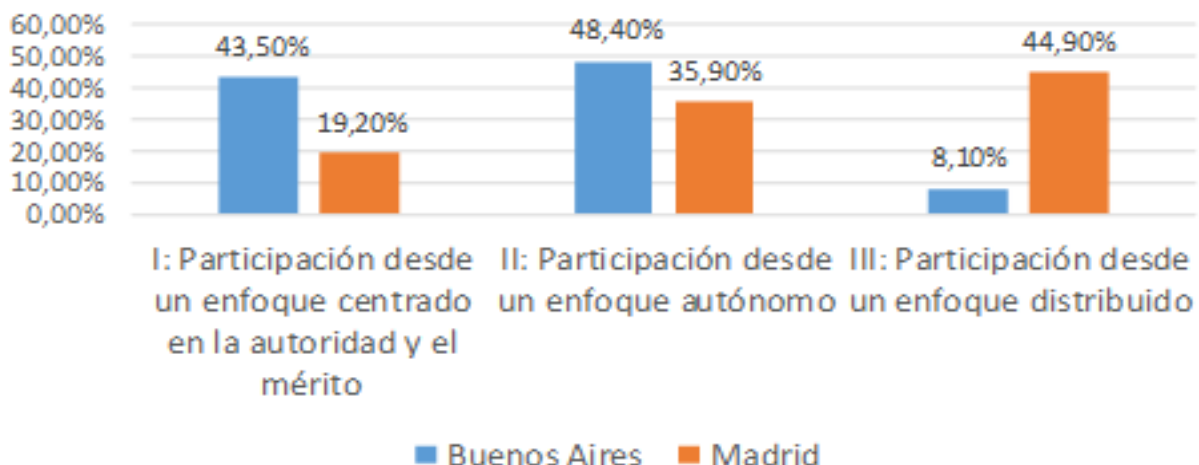

Figura 3. Niveles de participación por ciudad

Fuente: Elaboración propia. 


\section{Discusión y conclusiones}

Los resultados obtenidos en la muestra total de participantes indican que el alumnado en su mayoría tiene una representación sobre la participación desde un enfoque autónomo respecto al método para la elección de un delegado (nivel II), esto es, tiene criterios propios acerca de cómo tendría que ser el procedimiento democrático, independientemente de la medida adoptada por la profesora. En gran medida, las explicaciones en este nivel hacen alusión a mecanismos de voto, al igual que en el trabajo de Giné y otros (2014), donde se observa que, de forma genérica, el alumnado opta por el voto como una vía óptima para la toma de decisiones en su escuela.

Igualmente, los datos sugieren cómo algunas de estas concepciones pueden encontrarse en buena medida vinculadas a las propias experiencias escolares, lo cual en ocasiones puede llegar a constituir un obstáculo cuando lo que se ha experimentado han sido fundamentalmente prácticas escolares poco participativas y democráticas, sin ninguna posibilidad de llegar a trascenderlas o transformarlas. Y eso puede explicar en parte algunos de los argumentos dados por estudiantes cuando señalan que únicamente puede ejercer de delegada una persona durante todo el curso escolar, o que solo la profesora puede decidir, no existiendo una representación más justa socialmente sobre la participación desde un enfoque de justicia distribuida (nivel III), que implicaría una toma de decisiones más justa para cada alumno.

Esto guarda relación con los resultados obtenidos en el estudio de Barreiro (2013a) donde la representación distributiva de la justicia también fue la que menos estudiantes respondieron. Por ello, pensamos que a pesar de que vayan surgiendo ideas e iniciativas centradas en promover una participación más democrática en los centros educativos, todavía es necesario promover la construcción de una ciudadanía orientada hacia la justicia social por parte del alumnado (Burger, 2017; Coiduras et al., 2016; Jacott et al., 2019; Larkins, 2014; Percy-Smith, 2010, 2015; Thornberg y Elvstrand, 2012; Westheimer, 2015; Westheimer y Kahne 2004a, 2004b). Y esto implica la necesidad de dar una mayor relevancia al papel central que deben tener las escuelas como promotoras de procesos de participación ciudadana y democrática. El objetivo final es poder crear contextos educativos en los que se promueva el desarrollo de un conjunto de capacidades o competencias en el estudiantado, de forma que permita participar democráticamente, desarrollar un pensamiento crítico, expresar sus voces y tomar decisiones importantes que afecten a sus vidas en las escuelas y en su comunidad, desde una responsabilidad compartida por la justicia (Young, 2011). Si bien aún queda un largo recorrido para conseguir desarrollar auténticos procesos de construcción participativa en las escuelas, en donde chicas y chicos sean capaces de desarrollar por ejemplo una visión crítica frente a las situaciones de injusticia y desigualdad que se viven tanto en sus centros educativos, como en otros contextos tanto a nivel más local como global, ejerciendo la ciudadanía de una manera crítica, plena y autónoma por su parte.

Al igual que en otros estudios no encontramos diferencias significativas por edad (Barreiro, 2012, 2013a, 2013b; Helwig, 2002; Mager y Nowak, 2012; O’Hare et al., 2016). En este caso, resulta interesante destacar cómo en los dos grupos de edad, en estudiantes de $4^{\circ}$ y $6^{\circ}$ de Primaria, se encuentran los tres tipos de participación descritos. Resultados similares han sido encontrados en los estudios de Barreiro cuando se analizan los tres tipos de representaciones de justicia (Barreiro, 2013a), la creencia del mundo justo (Barreiro, 2013b), y las justificaciones que da el alumnado, del castigo (Barreiro, 2012). Lo 
que muestra que si bien a medida que aumenta la edad se producen argumentaciones más justas, distintos tipos de argumentaciones (menos o más justas) pueden estar presentes a lo largo del desarrollo. Y en concreto para la participación escolar, algunos estudios muestran que tampoco se ha encontrado diferencias significativas, por edades de los participantes (Mager y Nowak, 2012; Neff y Helwig, 2002; O’Hare et al., 2016). En nuestro caso, sería interesante ver en qué medida niñas y niños de 9 y 11 años utilizan o no diferentes tipos de argumentos cuando se enfrentan a distintos tipos de dilemas sociales.

En cuanto a las diferencias de género halladas en nuestro estudio, en donde las niñas se sitúan en una mayor proporción que los niños en el nivel III de participación, más cercano a la justicia social, los resultados concuerdan con los encontrados en distintos estudios con participantes de diferentes edades. Por ejemplo, en el estudio de Grayman y Godfrey (2013), donde las chicas adolescentes reflejaron una actitud hacia la justicia social, mayor que la de los chicos adolescentes, y en el de Sainz (2017), con relación a las concepciones de justicia social de docentes y alumnado de Educación Secundaria, más promotoras de dicha justicia en las chicas que en los chicos.

Otro elemento que puede estar vinculado a las diferencias de género encontradas, y que puede estar influyendo en sus representaciones de justicia social, se encuentra relacionado con el desarrollo de capacidades asociadas al género, en la medida en que se atribuye un mayor desarrollo de la empatía en las niñas, en contraste con los niños, y en consecuencia en la búsqueda por el bienestar de otras personas. Así pues, puede ser de gran relevancia indagar en las relaciones que pueden existir entre el desarrollo del comportamiento y razonamiento prosocial y las representaciones sobre la justicia social, teniendo en cuenta que algunos estudios han encontrado que las niñas y chicas adolescentes suelen tener conductas, pensamientos y respuestas afectivas más prosociales que sus compañeros chicos (Eisenberg, 2006; Jaffe y Hyde, 2000; Metzger, y Smetana, 2010).

$\mathrm{Al}$ contrario que en investigaciones anteriores (Mager y Nowak, 2012), en este estudio, sí encontramos diferencias significativas en cuanto a la ciudad de los participantes, siendo más justas las ideas que tiene el estudiantado sobre la participación escolar en Madrid frente a Buenos Aires. Estas diferencias por ciudades pensamos que pueden vincularse en parte al contexto sociocultural y socioeconómico en el que se encuentran los participantes de ambos países y al tipo de prácticas educativas y de tipo participativo promovidas en las escuelas. Por ejemplo, en este conurbano bonaerense, existen programas sociales que pueden estar reforzando los problemas de desigualdad, generando una intervención asistencial por parte del Estado, y una aceptación y reproducción de las injusticias por parte de la ciudadanía, por lo que se apunta a la necesidad de construir una ciudadanía de pleno derecho (Soldano, 2008).

En este sentido, Romero, Krichesky y Zacarías (2012), señalan la necesidad de revisar la justicia social en educación, desde la perspectiva tridimensional en Buenos Aires, a través de evaluaciones que aporten información de cuestiones socioculturales de las comunidades de cada escuela. Así, a partir de estas evaluaciones, se podrán poner en marcha variadas prácticas en educación contextualizadas para cada entorno y que, a su vez, tengan respaldo en la normativa vigente, remarcando la importancia de hacer que el alumnado bonaerense participe de forma activa, por ejemplo, en debates de cuestiones relacionadas con las injusticias sociales. De lo contrario, existe el riesgo de que, de alguna manera, el alumnado en contextos más desfavorecidos, donde no ha tenido apenas oportunidades de participar 
activa y democráticamente en la escuela, pueda llegar a situarse en una posición ciudadana más bien pasiva, sin cuestionarse que existan desigualdades y que tengan derecho a ser partícipes en la toma de decisiones importantes sobre sus vidas.

Asimismo, niñas y niños que comprenden mejor sus derechos suelen mostrar un mayor compromiso y participación en las actividades escolares (Burger, 2017; Covell, Howe y McNeil, 2008; Seguro, 2016), por lo que urge la necesidad de atender más detenidamente a cada contexto particular, y especialmente a los contextos más desfavorecidos. En este caso, se trata de niñas y niños que tienen menos acceso a recursos educativos, sociales y psicológicos, lo que puede impactar de forma negativa no solo en la percepción que tienen acerca de sus derechos de participación, sino también en el ejercicio de estos derechos. Por ejemplo, en cuanto al impacto que supone contar con escasos recursos psicológicos y sociales en la infancia, estudios recientes muestran que las evaluaciones realizadas por los niños acerca de su derechos de participación en la escuela se encuentran relacionados con el bienestar subjetivo, la percepción de no discriminación (es decir, un clima percibido de igual respeto por todos), la sensación de seguridad, y la disponibilidad de una persona de confianza en el ambiente escolar (Burger, 2017). Y muchos de estos elementos no se promueven de forma proactiva en las escuelas. Por otra parte, es posible que las niñas y niños que se desarrollan en contextos desfavorecidos tengan un menor nivel de conocimiento acerca de sus derechos, al mismo tiempo que de las situaciones de injusticia y desigualdad, lo que hace que tengan más dificultades para posicionarse de forma más activa y crítica frente a dichas situaciones, lo que les lleva a su vez a tener una menor participación en los distintos contextos en que viven.

En este estudio hemos planteado un dilema sobre una situación que puede resultar próxima a la realidad infantil, atendiendo a la relevancia que han señalado algunos autores de dotar de contexto a los ejemplos usados en las investigaciones con o adolescentes o niños (Barreiro, 2013a). He aquí, otra razón, por la que creemos que resultan diferencias significativas entre las ideas del alumnado en las dos ciudades: mientras que, en la mayoría de escuelas primarias en Madrid, es muy común la elección de representantes escolares, en las de Buenos Aires, parece ser que no cuentan con esta experiencia, al menos dentro de las escuelas primarias de este estudio.

Por ejemplo, cuando se les describe la función de los representantes de la clase, muchos estudiantes de Buenos Aires señalan las figuras de "abanderadas", "abanderados" y "escoltas" de la clase. Estas figuras hacen referencia a aquellas chicas y chicos elegidos por docentes, y en alguna ocasión también por sus iguales, pero siempre como un acto de alta consideración por sus buenas calificaciones, para portar la bandera del país. Por lo que en este caso se trata más bien de un acto escolar de tipo ceremonial, simbólico e identitario, y en donde la participación de chicas y chicos estudiantes es decidida por el profesorado en base al mérito académico.

Somos conscientes de que elegir a una persona representante de clase constituye solo un ejemplo de un pequeño acto de participación en la escuela y no puede ser considerado como participación democrática plena y absoluta por parte del alumnado en la toma de decisiones escolares. Además, como hemos comprobado, estas experiencias participativas ni siquiera están presentes en algunos contextos y realidades. A pesar de ello, vemos la necesidad de que se promuevan este tipo de prácticas en las escuelas, como un comienzo para a partir de ahí ir generando situaciones escolares cada vez más inclusivas con el objetivo de que podamos acercarnos a unas prácticas realmente democráticas y 
participativas que sean más justas y globales, entre todo el alumnado (Goodnight et al., 2019). Como, por ejemplo, proporcionando oportunidades reales para que niñas y niños puedan implicarse activamente en su propio aprendizaje a partir de prácticas y metodologías más inclusivas y participativas, promoviendo elecciones democráticas para elegir representantes y tomar decisiones sobre situaciones importantes en la vida escolar, potenciando una mayor participación de las familias en las escuelas. Al mismo tiempo que impulsando una mayor colaboración de estudiantes en el diseño del currículo y los temas a trabajar en clase, así como una mayor implicación de estudiantes y familias que provienen de contextos socialmente más desfavorecidos a través de acciones concretas, impulsando el trabajo por proyectos en clase, diseñando tareas que permitan pensar de forma crítica sobre las situaciones de injusticia y desigualdad y las condiciones estructurales que las producen o promoviendo un mayor reconocimiento e inclusión de las voces de estudiantes que pertenecen a colectivos minoritarios o que se encuentran en situación de desventaja social.

Estudiantes que no conocen la posibilidad de elegir a una compañera o compañero como representante de clase, mucho menos pensarán que pueden decidir sobre situaciones escolares por sí mismos, ni sobre situaciones de injusticias y desigualdades sociales. Por lo que es relevante, además, conocer las diferentes experiencias reales y concepciones sobre participación, entre estudiantes que tienen mayores oportunidades y experiencia en órganos o estructuras de participación frente a los que no la tienen (O’Hare et al., 2016; Pearce y Wood, 2019; Seguro, 2016).

Por otra parte, es importante destacar la idea de que solo podrán defender sus derechos, si son suficientemente conscientes de ellos (Burger, 2017), por lo que para promover una igualdad efectiva y una mayor cercanía a la justicia social es necesario que se escuchen sus voces, creando mecanismos directos para ellos, especialmente de aquellos que se encuentren en situaciones de desventaja social o escolar.

En definitiva, con este estudio pretendemos que se conciencie a la sociedad de lo crucial que es la participación del alumnado, aunque para promover la justicia social, no vale cualquier tipo de participación (Mager y Nowak, 2012). Para que esta sea real y no simbólica, debe reunir una serie de condiciones, desde un marco común de investigación. En primer lugar, debe partir de las voces del alumnado y enfocarse desde el diálogo con este. Además, debe considerar a niñas y niños como totalmente competentes, en el mismo plano que las personas adultas (Pearce y Wood, 2019) y ser comprendidos por ellas (Burger, 2017), dándoles un sentido de agencia (Cook-Shater, 2020) y de empoderamiento (Pearce y Wood, 2019), de forma que se trascienda las prácticas escolares justas a las prácticas sociales justas, o según nos indican algunos estudios, a la inversa, desde asociaciones de jóvenes, a la escuela (Horgan et al., 2017).

Es por ello que debemos estar dispuestas a cambiar de manera notable la forma de trabajar en las escuelas, y por extensión, en la sociedad (Pearce y Wood, 2019), atendiendo también por ejemplo al currículo oculto escolar tradicional, o a aquellas ideas más alejadas de la justicia social que aún mantienen muchos agentes educativos, ya que pueden estar influyendo negativamente a gran parte de la comunidad educativa, contrarrestando la intención formal de educar para un alumnado participativo y crítico hacia las situaciones de injusticia y desigualdad (Thornberg y Elvstrand, 2012).

Finalmente, queremos señalar que el presente estudio nos aporta datos que revelan cómo niñas y niños de 9 y 11 años son capaces de reflexionar sobre situaciones de justicia o 
injusticia hipotéticas, como las planteadas en el dilema sobre participación escolar. Al mismo tiempo que nos muestra cómo en esta tarea de razonamiento social son capaces de llegar a tener una visión crítica y emitir juicios de aceptación o rechazo a la autoridad, el mérito, las normas, la autonomía individual y la equidad. Este hecho nos hace pensar en la necesidad de seguir investigando en este ámbito con el objetivo de conocer con mayor profundidad cómo se desarrollan y coordinan estas ideas a través de distintas situaciones y contextos sociales, reales e hipotéticos, para interpretar y evaluar su realidad social y más específicamente para comprender la participación, toma de decisiones y justicia social.

\section{Referencias}

Barreiro, A. (2012) El desarrollo de las justificaciones del castigo: ¿Conceptualización individual o apropiación de conocimientos colectivos? Estudios de Psicología, 33(1), 67-77. https://doi.org/10.1174/021093912799803845

Barreiro, A. (2013a). The ontogenesis of social representation of justice: Personal conceptualization and social constraints. Papers on Social Representations, 22, 1-26.

Barreiro, A. (2013b). The appropriation process of the belief in a just world. Integrative Psychological and Behavioral Science, 47(4), 431-449. https://doi.org/10.1007/s 12124-013-9246-y

Bernard, H., Wutich, A. y Ryan, G. (2017). Analyzing qualitative data: Systematic approaches. Sage

Braun, V. y Clarke, V. (2006). Using thematic analysis in psychology. Qualitative Research in Psychology, 3, 77-101. https://doi.org/10.1191/1478088706qp063oa

Bruno, D. y Barreiro, A. (2015). La representación social de la democracia de adolescentes argentinos. Estudios de Psicología, 8(3), 33-40. https://doi.org/10.5231/psy.writ.2015.1506

Burger, K. (2017). The role of social and psychological resources in children's perception of their participation rights. Children and Touth Services Review, 79, 139-147. https://doi.org/10.1016/j.childyouth.2017.06.019

Campos, M. L. (2015). Las representaciones sociales y la reconstrucción de las prácticas educativas, un acercamiento a los sujetos. En A. Méndez (Coord.), Representaciones sociales en ámbitos educativos (pp. 188-219). Red Durando de Investigadores Educativos.

Coiduras, J. L., Balsells, M. A., Alsinet, C., Urrea, A., Guadix, I. y Belmonte, O. (2016). La participación del alumnado en la vida del centro: Una aproximación desde la comunidad educativa. Revista Complutense de Educación, 27(2), 437-456. https://doi.org/10.5209/rev_RCED.2016.v27.n2.46353

Convención de Naciones Unidas sobre los Derechos del Niño. (1989). Convención sobre los Derechos del Niño, adoptada por la Asamblea General de las Naciones Unidas el 20 de noviembre de 1989. Instrumento de ratificación del 30 de noviembre de 1990. Asamblea General de Naciones Unidas.

Cook-Sather, A. (2020). Student voice across contexts: Fostering student agency in today's schools. Theory into Practice, 59(2)182-191. https://doi.org/10.1080/00405841.2019.1705091

Covell, K., Howe, B. R., y McNeil, J. K. (2008). If there's a dead rat, don't leave it. Young children's understanding of their citizenship rights and responsibilities. Cambridge Journal of Education, 38(3), 321-339. https://doi.org/10.1080/03057640802286889

De Smul, M., Heirweg, S., Devos, G. y Van Keer, H. (2019). It's not only about the teacher! A qualitative study into the role of school climate in primary schools' implementation of selfregulated learning, School Effectiveness and School Improvement, 12, 381-404.

https://doi.org/10.1080/09243453.2019.1672758 
Dewey, J. (1916). Democracy and education: An introduction to the philosophy of education. MacMillan.

Eisenberg, N. (2006). Prosocial development. En W. Damon, R. M. Lerner y N. Eisenberg (Eds.), Handbook of child psychology. social, emotional, and personality development (pp. 646-718). John Wiley \& Sons.

Fraser, N. (2008a). Escalas de justicia. Herder.

Fraser, N. (2008b). La justicia social en la era de la política de identidad: Redistribución, reconocimiento y participación. Revista de Trabajo, 4(6), 83-99.

García-Pérez, D. y Montero, I. (2014). Organización y fomento de la participación del alumnado en educación primaria. Un estudio cualitativo de casos. Multidisciplinary Journal of Educational Research, 4(2), 211-238.

García-Vélez, T. (2016). Representaciones de la ciudadanía: Una visión cosmopolita. Estudio con estudiantes y profesores de Educación Secundaria (Tesis doctoral). Universidad Autónoma de Madrid, España.

Giné, S., Grau, V., Piñana, M. y Suñé, J. (2014). La responsabilidad en la participación: Un valor cooperativo en la educación primaria. Didáctica de las Ciencias Experimentales y Sociales, 28, 95-107. https://doi.org/10.7203/dces.28.3329

Goodnight, C. I., Whitley, K. G., Brophy-Dick, A. A. (2019). Effects of response cards on fourthgrade students' participation and disruptive behavior during language art lessons in an inclusive elementary classroom. Journal of Behavioral Education, 19(1), 57-69.

https://doi.org/10.1007/s 10864-019-09357-2

Granizo, L. (2011). El papel de la participación del alumnado en los Institutos de Enseñanza Secundaria (Tesis doctoral). Universidad Autónoma de Madrid, España.

Grayman, J. K. y Godfrey, E. B. (2013). Social justice attitudes and their demographic correlates among a nationally representative sample of U.S. adolescents. Social Justice Research, 26(4), 422-444. https://doi.org/10.1007/s11211-013-0196-9

Heikkila, A. S., Vuopala, E. y Leinonen, T. (2017). Design-driven education in primary and secondary school contexts. A qualitative study on teachers' conceptions on designing. Pedagogy and Education, 26(4), 471-483. https://doi.org/10.1080/1475939X.2017.1322529

Hidalgo, N. y Perines, H. (2018). Dar voz a los protagonistas: La participación estudiantil en el proceso de enseñanza-aprendizaje. Revista Educación, 42(2), 1-28.

https://doi.org/10.15517/revedu.v42i2.27567

Holdsworth, R. (2000). Schools that create real roles of value for young people. Prospects, 30, 349362. https://doi.org/10.1007/BFO2754058

Horgan, D., Forde C., Martin, S. y Parkes, A. (2017). Children's participation: Moving from the performative to the social. Children's Geographies, 15(3), 274-288. https://doi.org/10.1080/14733285.2016.1219022

Jacott, L., Maldonado, A., García, T., Sainz, V., Juanes, A., Seguro, V. y Agustín, S. (abril, 2016a). Desarrollo humano, bienestar y ciudadanía orientada a la justicia social. Simposio presentado en $2^{\circ}$ Congreso Latinoamericano para el Avance de la Ciencia Psicológica. Buenos Aires.

Jacott, L., Maldonado, A., Pérez-Manjarrez, E., Sainz, V., García-Vélez, T. Juanes, A., Fernández, A. y Agustín S. (mayo, 2016b). Social justice in education: Capabilities, well-being, and social justice-oriented citizens. Simposio presentado en la Jean Monnet Cice network conference: Education, citizenship and social justice: innovation, practices and research. Madrid. 
Jacott, L., Maldonado, A., García-Vélez, T., Pérez Manjarrez, E., Sainz, V., Juanes, A., Fernández, A., Seguro, V., Agustín, S. y Plascencia, M. (2019). Social justice in education: Capabilities, well-being, and social justice-oriented citizens. En L. Jacott, T. García-Vélez y V. Seguro Gómez (Eds.), Education, citizenship and social justice: Innovation, practices and research (pp. 91100). CiCea-UAM

Jaffe, S. y Hyde, J. S. (2000). Gender differences in moral orientation: A metanalysis. Psychological Bulletin, 126(5), 703-726. https://doi.org/10.1037/0033-2909.126.5.703

Juanes, A. (2018). Representaciones de Justicia Social en estudiantes de Educación Primaria. Estudio cuantitativo y cualitativo comparado entre España y Argentina (Tesis doctoral). Universidad Autónoma de Madrid, España.

Juanes, A., Pérez-Manjarrez, E. y Fernández-González, A. (2013). Social justice representations of primary students. En P. Cunningham (Ed.) Identities and citizenship education: Controversy, crisis and challenges (pp. 511-515). CiCe.

Koster, M., Jan Pijl, S., Nakken, H. y Van Houten, E. (2010). Social participation of students with special needs in regular primary education in the Netherlands. International Journal of Disability, Development and Education, 57(1), 59-75. https://doi.org/10.1080/10349120903537905

Larkins, C. (2014). Enacting children's citizenship: Developing understandings of how children enact themselves as citizens through actions and acts of citizenship. Childhood 21(1), 7-21. https://doi.org/10.1177/0907568213481815

Lodge, C. (2005). From hearing voices to engaging in dialogue: Problematising student participation in school improvement. Journal of Educational Change, 6, 125-146. https://doi.org/10.1007/s10833-005-1299-3

Mager, U. y Nowak, P. (2012). Effects of student participation in decision making at school. A systematic review and synthesis of empirical research. Educational Research Review, 7, 3861. https://doi.org/10.1016/j.edurev.2011.11.001

Melton, G. B. (1980). Children's concepts of their rights. Journal of Clinical Child Psychology, 9(3), 186-190. https://doi.org/10.1080/15374418009532985

Metzger, A. y Smetana, J. G. (2010) Social cognitive development and adolescent civic engagement. En L. R. Sherrod, J. Torney-Purta, y C. A. Flanagan. (Eds.), Handbook of research on civic engagement in youth (pp. 221-248). John Wiley y Sons.

https://doi.org/10.1002/9780470767603.ch9

Miller, D. (1999). Principles of social justice. Harvard University Press.

Murillo, F. J. y Hernández, R. (2011) Hacia un concepto de justicia social. REICE. Revista Iberoamericana sobre Calidad, Eficacia y Cambio en Educación, 9(4), 8-23.

Neff, K. D. y Helwig, C. C. (2002). A constructivist approach to understanding the development of reasoning about rights and authority within cultural contexts. Cognitive Development 17(3), 1429-1450. https://doi.org/10.1016/So885-2014(02)00126-O

Nucci, L. P. (2001). Education in the moral domain. Cambridge University Press. https://doi.org/10.1017/CBO9780511605987

Nucci, L. P., Turiel, E. y Roded, A. (2018). Continuities and discontinuities in the development of moral judgments. Human Development, 60(6), 279-341. https://doi.org/10.1159/000484067

O’Hare, L., Santin, O., Winter, K. y McGuinness, C. (2016). The reliability and validity of a child and adolescent participation in decision-making questionnaire. Child: Care, Health, and Development, 42(5), 692-698. https://doi.org/10.1111/cch.12369 
Ochaíta, E. y Espinosa, M. (2004). Hacia una teoría de las necesidades infantiles y adolescentes. Necesidades y derechos en el marco de la Convención de Naciones Unidas sobre derechos del niño. McGraw-Hill.

Percy-Smith, B. (2010). Councils, consultations and community: Rethinking the spaces for children and young people's participation. Children's Geographies, 8, 107-122.

Percy-Smith, B. (2015). Negotiating active citizenship: young people's participation in everyday spaces. En K. Kallio, S. Mills y T. Skelton (Eds.), Politics, citizenship and rights. Geographies of children and young people. Springer. https://doi.org/10.1080/14733281003691368

Piaget, J. (1932). El juicio moral en el niño. Francisco Beltrán.

Pearce, T. C. y Wood, B. E. (2019) Education for transformation: An evaluative framework to guide student voice work in schools. Critical Studies in Education, 60(1), 113-130. https://doi.org/10.1080/17508487.2016.1219959

Rawls, J. (1971). A theory of justice. Oxford University Press.

Robles, P. (2015). El proyecto de autonomía en la institución escolar de la educación primaria. En A. Méndez (Coord.), Representaciones sociales en ámbitos educativos (pp. 220-242). Red Durando de Investigadores Educativos.

Romero, C., Krichesky, G. J. y Zacarías, N. (2012). Problemas de justicia social en el contexto educativo argentino: El caso del nivel secundario. Revista Internacional de Educación para la Justicia Social, 1(1), 94-110.

Ros, I. (2009). La implicación del estudiante con la escuela. Revista de Psicodidáctica, 14(1), 79-92.

Rudduck, J. y Fielding, M. (2006). Student voice and the perils of popularity. Educational Review, 58, 219-231. https://doi.org/10.1080/00131910600584207

Sainz, V. (2017). Representaciones de la justicia social en profesores y estudiantes de educación secundaria (Tesis doctoral). Universidad Autónoma de Madrid, España.

Sales, A., Moliner, O., Amiama, J. F. y Lozano, J. (2018). Escuela incluida. Recursos y estrategias para la participación ciudadana. Revista Mexicana de Investigación Educativa, 22(77), 433-458.

Seguro, V. (2016). Con voz: Derechos de participación y autonomía en la niñez (Tesis doctoral). Universidad Autónoma de Madrid, España.

Soldano, D. (2008). Vivir en territorios desmembrados. Un estudio sobre la fragmentación socioespacial y las políticas sociales en el área metropolitana de Buenos Aires (1990-2005). En A. Ziccardi (Comp.), Procesos de urbanización de la pobreza y nuevas formas de exclusión social: Los retos de las políticas sociales de las ciudades latinoamericanas del siglo XXI (pp. 37-69). Siglo del Hombre.

Susinos, T. y Rodríguez-Hoyos, C. (2011). La educación inclusiva hoy. Reconocer al otro y crear comunidad a través del diálogo y la participación. Revista Interuniversitaria de Formación del Profesorado, 25(1), 15-30.

Thornberg, R. y Elvstrand, H. (2012). Children's experiences of democracy, participation, and trust in school. International Journal of Educational Research, 53, 44-54. https://doi.org/10.1016/j.ijer.2011.12.010

Turiel, E. (2002). The culture of morality: Social development, context, and conflict. Cambridge University Press. https://doi.org/10.1017/CBO9780511613500

Westheimer, J. (2015). What kind of citizen? Educating our children for the common good. Teachers College Press. 
Westheimer, J. y Kahne, J. (2004a). What kind of citizen? The politics of educating for democracy. American educational research journal, 41(2), 237-269. https://doi.org/10.3102/00028312041002237

Westheimer, J. y Kahne, J. (2004b). Educating the "good” citizen: Political choices and pedagogical goals. Political Science and Politics, 37(2), 241-247. https://doi.org/10.1017/S1049096504004160

Young, I. M. (2011). Responsabilidad por la justicia. Morata.

\title{
Breve CV de las autoras
}

\begin{abstract}
Almudena Juanes
Orientadora en la Comunidad de Madrid y profesora del Departamento de Psicología Evolutiva y de la Educación de la Facultad de Formación de Profesorado y Educación de la Universidad Autónoma de Madrid. Doctora en Educación y Licenciada en Psicología, su labor docente se ha desarrollado también en las universidades: Nebrija, Alfonso X El Sabio y Universidad a Distancia de Madrid. Sus intereses de investigación fundamentales son: Justicia Social, representaciones de estudiantes y docentes en todas las etapas educativas, orientación educativa, psicología evolutiva y del desarrollo, razonamiento prosocial, alumnos con necesidades específicas de apoyo educativo, didáctica y organización en el aula. ORCID ID: https://orcid.org/0000-0002-3024-0705. Email: almudena.juanes@educa.madrid.org
\end{abstract}

\section{Liliana Jacott}

Profesora de la Facultad de Formación de Profesorado y Educación de la Universidad Autónoma de Madrid. Tiene una amplia experiencia en investigación y docencia en el campo de la psicología del desarrollo y la educación. En los últimos años, sus líneas de investigación se han centrado en las representaciones sobre la justicia social, educación para la ciudadanía, desarrollo humano y justicia social. Actualmente es Coordinadora del Área de Investigación de la Cátedra UNESCO de Educación para la Justicia Social de la UAM y Coordinadora de la línea de investigación "Representaciones sobre la justicia social" dentro de la Cátedra UNESCO de la UAM. ORCID ID: https://orcid.org/oooo0002-5826-3693.Email: liliana.jacott@uam.es 